\title{
NEW ALGORITHMIC ALTERNATIVES FOR THE OD MATRIX ADJUSTMENT PROBLEM ON TRAFFIC NETWORKS *
}

\author{
Esteve Codina, \\ Universidad Politècnica de Catalunya, Facultat de Matemàtiques i Estadística. \\ c/ Pau Gargallo, 5, 08028 Barcelona, España. esteve.codina@upc.es \\ Ricardo García, \\ Universidad de Castilla-La Mancha. E. U. Politécnica de Almadén. \\ Plaza Manuel Meca, 1. 13.400-Almadén, Ciudad Real, España.rgarcia@pol-al.uclm.es \\ Angel Marín. \\ Universidad Politécnica de Madrid. E. T. S. I. Aeronáuticos. \\ Plaza Cardenal Cisneros, 3. 28.040, Madrid, España. amarin@dmae.upm.es
}

January 10, 2002

\section{EXTENDED ABSTRACT}

\section{Introduction.}

For demand estimation on traffic networks, a source of information of relatively moderate cost are traffic counts on a subset of links of the network and this fact has motivated the development of bilevel mathematical programming formulations for adjusting O-D trip matrices that reproduce the observed traffic volumes. In the algorithmic field several methods have been developed specifically for the problem that, although can be viewed as heuristics, adopt approaches common to the general methods in optimization. Many of these methods can be viewed as a descent direction methods because in a step of the algorithm, an heuristic approximation of the steepest descent direction for the upper level objective function is evaluated. This paper compares some of the existing methods for the OD matrix adjustment problem, such as the method of Spiess in [7], the method of Chen in [2], the method of Yang in [8], and the method of Codina and Barceló in [3] with two new algorithmic alternatives developed by the authors. The first alternative is an adaptation of the proximal point algorithm that is based on a development of Codina in [4] for the approximation of the steepest descent direction of the upper level function and the second alternative developed by García and Marín in [5] consists in the linearization at each iteration of the lower level problem.

\section{Some methods for the O-D matrix adjustment problem}

Definitions and notation. The OD matrix adjustment problem (DAP) is a bilevel problem, where at upper level the demand is optimized taking account the traffic assignment problem (TAP) at lower level. Some of the methods described in this paper can be used for general asymmetric TAP whereas others can only be applied for additive and separable TAP. For a non negative O-D matrix $g$, let $\mathcal{V}(g)$ the set of TAP feasible link

\footnotetext{
${ }^{*}$ Research supported under Spanish CICYT project TRA99-1156-C02-01/2
} 
flows and let $\boldsymbol{\Omega}$ denote the pairs $(v, g)$ so that $g \geq 0$ and $v \in \mathcal{V}(g)$. Let $V(g)$ the optimal value of the objective function $T(v)=\sum_{a \in \mathcal{A}} \int_{0}^{v_{a}} C_{a}(x) d x$, of the additive and separable TAP for a given O-D matrix $g$. The function $V(g)=T\left(v^{*}(g)\right)$ is convex and differentiable.

In general if the assignment map $v^{*}(g)$, or solution set of the general asymmetric TAP provides unique solutions in the link flows, then the DAP can be formulated as:

$$
\text { Min }_{g \geq 0} F(g)=z_{1} F_{1}\left(v^{*}(g)\right)+z_{2} F_{2}(g)
$$

It must be remarked that the upper level function $F(g)$ is nondifferentiable. Usually $F_{1}(v)=\frac{1}{2}(v-\hat{v})^{\top} U(v-\hat{v})$ and $F_{2}(g)=\frac{1}{2}(g-\bar{g})^{\top} B(g-\bar{g})$ or an entropy function. $\hat{v}$ and $\bar{g}$ are the observed traffic and the reference demand.

A survey of existing methods and models until 1997 can be found in Barceló [1].

Spiess' method. It assumes that the equilibrium link flows $v^{*}(g)$ present a partial derivative $\frac{\partial v_{a}(g)}{\partial g_{\omega}}$ that can be approximated by the link use proportions $p_{a \omega}(g)$, so the gradient of the upper level function $F$ can be approximated by: $\nabla F \approx P_{1}^{\top} U\left(v_{1}-\hat{v}_{1}\right)=$ $d_{\mathrm{P}}$. At each iteration the new O-D matrix is taken by $g_{\omega}^{\ell+1}=g_{\omega}^{\ell}\left(1-\lambda d_{\mathrm{P}}\left(g^{\ell}\right)\right)_{\omega}$ where $\lambda$ is a step length that approximately minimizes $F(\cdot)$ along the direction $-d_{S}$, being $\left(d_{S}\right)_{\omega}=g_{\omega} \cdot\left(d_{P}\right)_{\omega}$. Clearly a steepest descent method would be obtained if the iteration would take the form $g_{\omega}^{\ell+1}=g_{\omega}^{\ell}-\lambda\left(d_{\mathrm{P}}\right)_{\omega}$.

Yang's method. Yang proposes an algorithm in which at each iteration the following problem is solved:

$$
\begin{array}{cl}
\operatorname{Min}_{v \geq 0, g \geq 0} & F^{(\ell}(g)=z_{1} F_{1}(v)+z_{2} F_{2}(g) \\
\text { s.t. } & v=v^{*}\left(g^{\ell}\right)+Z\left(g-g^{\ell}\right)
\end{array}
$$

The equilibrium link flows $v^{*}(g)$ for the O-D matrix $g$ are approximated linearly by the influence factors matrix $Z$. The new point is the solution $g^{\ell+1}$ of problem (2).

Chen's method. Chen's algorithm is a descent direction type heuristic method to solve DAP is: At iteration $\ell$ let $g^{(\ell}$ be the OD matrix evaluated in the previous iteration. Let $b=B\left(g^{(\ell}-\tilde{g}\right)+d_{\mathrm{Y}}^{(\ell}$, where $d_{\mathrm{Y}}^{\ell}$ is the Yang's direction as defined by problem (2). A direction $-d_{\text {Chen }}^{(\ell}=x^{\ell}-g^{(\ell}$ is defined, where $x_{\omega}^{(\ell}=2 g_{\omega}^{(\ell}$ if $b_{\omega}<0, x_{\omega}^{(\ell}=g_{\omega}^{(\ell}$ if $b_{\omega}=0$ and $x_{\omega}^{(\ell}=0$ if $b_{\omega}>0$. A step length $\lambda$ along $d_{\text {Chen }}^{(\ell}$ is evaluated and $g^{(\ell+1}=g^{(\ell}-\lambda d_{\text {Chen }}^{\ell}$. Finally, the OD matrix $g^{(\ell+1}$ is assigned keeping track of the path proportions $p_{a \omega}$ and the Yang direction, $d_{\mathrm{Y}}^{\ell+1}$ is evaluated.

\section{Adaptation of the proximal point algorithm}

The proximal point algorithm solves DAP defining a sequence of problems of the type $\operatorname{Min}_{g} F(g)+\frac{\rho_{k}}{2}\left\|g-g_{k}\right\|_{2}^{2}$ for a sequence $\left\{\rho_{k}\right\}$ of parameters, $\rho_{k} \rightarrow \infty$, for a convex function $F$. The sequence of solutions $g_{k}$ converge to a minimum of $F$. In our case $F(g)=F_{1}\left(v^{*}(g)\right)$ and the problem will be approximately solved by means of an exact penalty approach. A reformulation of DAP is:

$$
\begin{aligned}
\operatorname{Min}_{(v, g) \in \boldsymbol{\Omega}} & F_{1}(v)+\frac{\rho_{k}}{2}\left\|g-g_{k}\right\|_{2}^{2} \\
\text { s.t. } & T(v)-V(g)=0
\end{aligned}
$$


It must be noted that the function $T(v)-V(g)$ is always nonnegative on the cone $\boldsymbol{\Omega}$, greater than zero if $v \neq v^{*}(g)$, and zero otherwise. If the constraints are penalized, then (3) can be approximated by:

$$
\operatorname{Min}_{(v, g) \in \boldsymbol{\Omega}} \quad \psi(v, g)=T(v)-V(g)+z_{k} F_{1}(v)+\frac{z_{k} \rho_{k}}{2}\left\|g-g_{k}\right\|_{2}^{2}
$$

where $z_{k} \cdot \rho_{k} \rightarrow 0$. It can be shown that problem (4) consistently approximates problem (3). This is shown in Codina [4] where a very similar scheme is followed but for the purpose of approximating the gradient of the upper level objective function. In order to solve problem (4), the partial linearization of Patriksson [6] is applied. At iteration $\ell$, of the partial linearization algorithm, the objective function $\phi^{(\ell}(v, g)$ of the resulting subproblem at point $\mathbf{g}^{\ell}$ is $\phi^{(\ell}(v, g)=T(v)-g^{\top} C^{(\ell}+z_{k} F_{1}(v)+\frac{z_{k} \rho_{k}}{2}\left\|g-g_{k}\right\|_{2}{ }^{2}$, where $C^{(\ell}=C^{*}\left(\mathbf{g}^{\ell}\right)$ are the O-D travel times resulting from the assignment of the O-D matrix $\mathrm{g}^{\ell}$. The convergence of the partial linearization algorithm for problem (4) can be found in Codina [4].

\section{Upper approximation method}

García and Marín [5] proposed this method to obtain simultaneously the OD matrix and the parameters of a logit model of a combined TAP. We develop an adaptation of the upper approximation method (UAM) for DAP. UAM consists of solving a sequence bilevel models obtained by replacing the lower problem by an easier solvable problem. More formally, consider that the point $g^{\ell}$ is the iterate at the iteration $\ell$. $\operatorname{Set} \operatorname{VIP}\left(C, \mathcal{V}\left(g^{\ell}\right)\right)$ the lower problem. Considering at $\ell$ an approach of the function $C(v, g)$ at $g^{\ell}, C^{\ell}(v, g)$. The UAM next iteration is defined by $g^{\ell+1} \in \operatorname{Arg} \min F(v, g), \forall v \in \mathcal{V}^{\ell}(g)$, where $\mathcal{V}^{l}(g)$ is the set of the optimal solutions of $\operatorname{VIP}\left(C^{\ell}, \mathcal{V}(g)\right.$ : Find $v \in \mathcal{V}(g)$ satisfying $C^{\ell}(v, g)\left(v^{\prime}-v\right) \geq$ $0, \forall v^{\prime} \in \mathcal{V}(g)$.

The choice of the mapping $C^{\ell}$ must be effected so that the point-set mapping $\mathcal{V}^{\ell}$ can be explicitly computed. In general, we assume that the approximation of $C^{\ell}$ takes a constant cost associated with the of link costs.

An approximation of the cost mapping can be derived from choosing $C^{\ell}(v, g)=$ $C\left(v^{*}\left(g^{\ell}\right), g^{\ell}\right)$, where $v^{*}\left(g^{\ell}\right)$ denotes the (unique) optimal variable of the lower problem.

This approximation is a linear one of the link cost mapping at the equilibrium for the O-D matrix $g^{\ell}$ given, and it does not depend on the link flow (it is constant). The optimal paths can be computed for the approximated lower problem in this case, and the cost of these paths are independent of their flows. Therefore, if the components associated with non optimal paths are equal to zero of a path flow $h$, then it is an optimal flow for the approximated lower problem.

The approximation holds the property

$$
v^{*}(g) \in \mathcal{V}^{\ell}(g), \quad \text { for all } g \in U^{\ell}
$$

for some neighborhood $U^{\ell}$ of the point $g^{\ell}$, and it motivates the name UAM.

In order to describe the set $\mathcal{V}^{\ell}(g)$, we introduce the sets $\mathcal{R}_{\omega}^{\ell}=\left\{r \in \mathcal{R}_{\omega} \mid C_{r}\left(g^{\ell}\right)=C_{\omega}^{*}\left(g^{\ell}\right)\right\}$ and $\mathcal{R}^{\ell}=\cup_{\omega} \mathcal{R}_{\omega}^{\ell}$, where $C_{r}\left(g^{\ell}\right)$ is the cost of the path $r$ at the equilibrium for the given matrix $g^{\ell}$, and $C_{\omega}^{*}\left(g^{\ell}\right)$ is the equilibrium cost for the pair OD $\omega$.

The set $\mathcal{V}^{\ell}$ can be expressed as

$$
\mathcal{V}^{\ell}(g)=\left\{v \mid v=\Delta h, \text { for some } h \in \mathcal{H}(g) \cap \mathcal{V}_{0}^{\ell}\right\}
$$


where the following set is considered $\mathcal{V}_{0}^{\ell}=\left\{h \mid h_{r}=0, \quad\right.$ for all $\left.r \in \mathcal{R}-\mathcal{R}^{\ell}\right\}, \Delta$ is the link-route incidence matrix and $\mathcal{H}(g)$ is the set of feasible path flow for the OD matrix $g$.

Even in the case that the point-set mapping $\mathcal{V}^{\ell}(g)$ is known, it may be necessary to describe all the paths with positive flow at the equilibrium for the variable $g^{\ell}$. By this reason, may be introduced the selection functions to simplify $\mathcal{V}^{\ell}(g)$. These approaches replace the constraint $v \in \mathcal{V}^{\ell}(g)$ by

$$
v=\Phi^{\ell}(g) \in \mathcal{V}^{\ell}(g) \forall g
$$

If we look at the expression of $\mathcal{V}^{\ell}(g)$ in (5) this selection function consists of defining a function which gives the path flow in function of the variable $g$, that is

$$
h=\phi^{\ell}(g) \text { where } h \in \mathcal{H}(g) \cap \mathcal{V}_{0}^{\ell},
$$

thus, the selection function becomes

$$
v=\Phi^{\ell}(g)=\Delta \phi^{\ell}(g) .
$$

Remark that the selection function is defined by a rule to assign the variable $g$ to the optimal paths at the iteration $\ell$.

Yang's algorithms are defined by setting of a specific selection function (6).

\section{$5 \quad$ Numerical results}

It has been carried out a comparison between the previous heuristic approaches on small size networks in order to evaluate the computational costs, the convergence and the quality of the solution. The networks used have been taken from the references of Codina and Barceló [3] and Yang [8]

\section{Conclusions}

Two new algorithmic alternatives are presented to solve the OD matrix adjustment problem on traffic networks: a new adaptation of the proximal point and a new class of upper approximation algorithms. Both algorithms are considered in relation with the state of art of OD matrix adjustment algorithms. Some computational tests comparing the new and the state of art algorithms are presented.

\section{References}

[1] Barceló J. (1997) "A Survey of Some Mathematical Programming Models in Transportation". Top, Vol. 5, No.1 June 1997. pp. 1-40.

[2] Chen Y. "Bilevel Programming Problems: Analysis, Algorithms and Applications" Ph. D. dissertation, Départament d'Informatique et de Recherche Opérationelle, Université de Montréal. 1994

[3] Codina E., Barceló J. (2000) "Adjustment of O-D trip matrices from traffic counts: an algorithmic approach based on conjugate directions". Proceedings of the $8^{\text {th }}$ EWGT. Rome, July 2000. pp. 427-432. 
[4] Codina E. (2002) "Consistency of an approximation to the upper level objective function gradients in the o-d demand adjustment problem". Research Report DR/2002-01. (Revised version) EIO-UPC

[5] García, R. and Marín, A. (1998) "A bilevel programming approach for estimation of origin-destination matrix and calibration of parameters of a network equilibrium model with combined modes". Proceedings of the $6^{\text {th }}$ EWGT. Gothenburg, 1998.

[6] Patriksson M. (1993) "Partial linearization Methods in Nonlinear Programming ". Journal of Optimization Theory and Applications, 78, pp. 227-246

[7] Spiess, H. (1990). "A Gradient Approach for the O-D Matrix Adjustment Problem". Centre de Recherche sur les Transports de Montral. Publication 693, May 1990.

[8] Yang H. (1995) "Heuristic Algorithms for the Bilevel Origin-Destination Matrix Estimation Problem". Trans. Res. B Vol 29B No. 4, pp 231-242 